\title{
Método de incorporación estratégica de tecnología biomédica para instituciones de salud
}

\author{
M.A. Ardila ${ }^{1}$, A. Gómez ${ }^{1}$, J.E Camacho-Cogollo ${ }^{2}$ \\ ${ }^{1}$ Estudiante ingeniería biomédica, Universidad EIA, Medellín, Colombia \\ ${ }^{2}$ Docente Investigador, Universidad EIA, Medellín, Colombia
}

\begin{abstract}
Resumen-Los costos continuos, altos e innecesarios de la tecnología, la escasa atención del paciente, las decisiones tomadas por personas con poca experiencia y el desperdicio de recursos públicos dedicados a la salud, generan la necesidad de desarrollar un proceso racional y sistemático para la incorporación de equipos médicos. El objetivo de este trabajo fue hacer que un método comience con una recopilación de información a través de una revisión de la literatura y la implementación de una encuesta para diagnosticar el estado de los procesos de incorporación en diferentes instituciones de salud y saber cuáles son las etapas críticas y los pasos a seguir en cada uno de ellos. El método se implementó en un caso real con la adquisición de dos tecnologías: el acelerador lineal y un esterilizador de vapor. El método fue validado con dos expertos responsables de la adquisición de estos equipos para determinar su usabilidad e importancia en el proceso de incorporación de la tecnología biomédica. La validación mostró resultados cuantitativos y positivos para ambas tecnologías, ya que los expertos estuvieron satisfechos con cada uno de los aspectos evaluados y el informe final proporcionado por el método.
\end{abstract}

Palabras Clave - Tecnología biomédica; incorporación; tecnología; adquisición.

\section{Method of Strategic Incorporation of Biomedical Technology for HEALTH InSTITUTIONS}

\footnotetext{
Abstract - The continued high and unnecessary costs of technology, poor patient care, decisions made by people with little experience and waste of public resources devoted to health. All generate a need to develop a rational and systematic process for the incorporation of medical equipment. The aim of this work was to create such a method through collection of information, a literature review and implementation of a survey to diagnose the status of the processes of incorporation into different healthcare institutions and learn the critical stages and steps to be performed in each. The method was implemented in a real case with the acquisition of two technologies, a linear accelerator and a steam sterilizer. The method was validated with two experts responsible for the acquisition of this equipment to determine its usability and importance in the process of incorporation of biomedical technology. Validation showed quantitative and positive results for both technologies because the experts were satisfied with each of the aspects evaluated and the final report provided by the method.
}

Keywords - Biomedical technology; Incorporation; Technology assessment; Acquisition.

\% Dirección para correspondencia: Javier.camacho@eia.edu.co

DOI: https://doi.org/10.24050/19099762.n20.2017.1071 


\title{
MÉTODO DE INCORPORAÇÃO ESTRATÉGICA DE TECNOLOGIA BIOMÉDICA PARA INSTITUIÇÕES DE SAÚDE
}

\begin{abstract}
Resumo-Os altos e desnecessários custos continuados da tecnologia, a escassa atenção aos pacientes, as decisões tomadas por pessoas com pouca experiência e o desperdício de recursos públicos dedicados à saúde, geram a necessidade de desenvolver um processo racional e sistemáticos para a incorporação de equipamentos médicos. Os objetivos deste trabalho foram fazer um método que comece com uma compilação de informação através de uma revisão da literatura e a implementação de uma sondagem para diagnosticar o estado dos processos de incorporação nas diferentes instituições de saúde e saber quais são as etapas críticas e os passos a seguir em cada um deles. O método implementou-se num caso real com a aquisição de duas tecnologias, o acelerador linear e um esterilizador de vapor. Este foi validado com dois peritos responsáveis da aquisição destes equipamentos para determinar sua usabilidade e importância no processo de incorporação da tecnologia biomédica. A validação mostrou resultados quantitativos e positivos para ambas tecnologias, já que os peritos estiveram satisfeitos com cada um dos aspectos avaliados e o relatório final proporcionado pelo método.
\end{abstract}

Palavras-chave - Tecnologia biomédica; Incorporação, Auditoria tecnológica; Aquisição.

\section{INTRODUCCIÓN}

$\mathrm{E}^{1}$ alto nivel de desarrollo de los dispositivos biomédicos convierte a los centros de salud en espacios tecnológicamente avanzados, haciendo de los equipos médicos herramientas indispensables para resolver problemas de la salud humana. Se estima que el cuidado de la salud dependerá del desarrollo, disponibilidad y adquisición de las nuevas tecnologías, todo esto a través de una correcta evaluación y gestión de la tecnología. La incorporación de tecnología (IT) hace parte del macro proceso de gestión de la tecnología, esta incluye subprocesos de planeación y adquisición tecnológica adecuados, sustentados en evitar gastos innecesarios que pongan en riesgo el sistema de salud y las finanzas de las instituciones de salud [1-5].

La evaluación de la tecnología, la cual es la primera etapa del proceso de incorporación de tecnología, es un proceso que necesita del trabajo de expertos en diversas disciplinas para sintetizar información relativa a las cuestiones médicas, sociales, económicas y éticas relacionadas con el uso de la tecnología sanitaria de manera sistemática, transparente, imparcial y robusta [4].

El método propuesto en este trabajo busca cuantificar los resultados de los procesos realizados en una incorporación de tecnología (IT) por medio del diseño de formatos que tengan en cuenta asuntos importantes como seguridad, beneficios clínicos, necesidades, aspectos económicos, éticos-sociales y servicios de los proveedores $[4,7]$. Se asigna el peso en porcentaje a cada criterio para tomar una decisión con base en los resultados numéricos y así ajustar el presupuesto disponible de cada institución y dirigir todos sus procesos al cumplimiento de las metas proyectadas a futuro [8].

\section{Metodología}

El estudio se desarrolla en cuatro fases: la primera consiste en una revisión bibliográfica de los trabajos académicos, comparando los métodos de incorporación en un contexto mundial. Los resultados sentaron las bases para la construcción de una encuesta a diferentes ingenieros clínicos de Colombia, lo que permitió definir las etapas necesarias para la elaboración de una propuesta metodológica. En la siguiente fase se construyó la propuesta describiendo los formatos asociados y la guía para su uso. En la tercera fase se implementa el método a través de dos casos de incorporación de equipo médicos y, por medio de la evaluación de dos expertos, se mide su posible impacto. Por último se analizan los resultados obtenidos y se determina el grado de usabilidad, importancia e impacto del método propuesto en el ahorro de recursos para las instituciones de salud.

\section{Resultados}

Los resultados de este desarrollo se presentarán en la secuencia de fases como se establece en la metodología.

\section{A. Análisis de la encuesta}

Para el desarrollo de la encuesta se usó la herramienta de diseño de encuesta Qualtrics, la cual genera un análisis de resultados de forma gráfica. La encuesta se envió a 25 ingenieros clínicos (IC) de Instituciones Prestadoras de Servicios (IPS) de Colombia.

La encuesta entregó resultados que permitieron generar un diagnóstico sobre la implementación de incorporación estratégica de tecnología en ese país. De la muestra seleccionada se destaca que solo el $68 \%$ de las instituciones de salud tienen estructurado un comité multidisciplinario como parte del proceso de IT y solo un $26 \%$ de los pro- 
fesionales están satisfechos con el proceso. Así mismo se detectó una falencia en la priorización en IT, puesto que se evidenciaron malas prácticas en la identificación de necesidades, y generalmente los equipos obsoletos o con historial de fallas no se reemplazan. Esto se complementa con el hecho de que un $47 \%$ de las IPS no cuentan con un plan de IT con proyección en el tiempo, generando una percepción de poca planeación estratégica para los planes de compra y los presupuestos económicos.

Por otro lado los, IC indicaron que en las consideraciones más relevantes para un adecuado proceso de IT se deberían contemplar aspectos de técnicos de la tecnología, además de variables como la seguridad del paciente, adecuaciones de infraestructura, beneficios clínicos, patologías a tratar y por último aspectos éticos/sociales. La Fig.
2 ilustra en su orden el nivel de importancia que definieron los IC durante la encuesta.

Para los profesionales en IC, los aspectos de mayor importancia para evaluar a un proveedor de tecnología biomédica son: la capacidad técnica y disponibilidad de repuestos y accesorios, acompañada de los tiempos de garantía que este ofrezca. Además se tienen en cuenta valoraciones de la calidad, tiempo de entrega de los productos, formas de pago, referencia de otras instituciones y experiencia en el mercado. Así mismo se identificó que para las IPS es determinante que el producto no tenga reportes de alertas a nivel nacional o internacional, las condiciones de garantía así como los tiempos de respuesta ante llamados de solicitudes de servicio técnico.

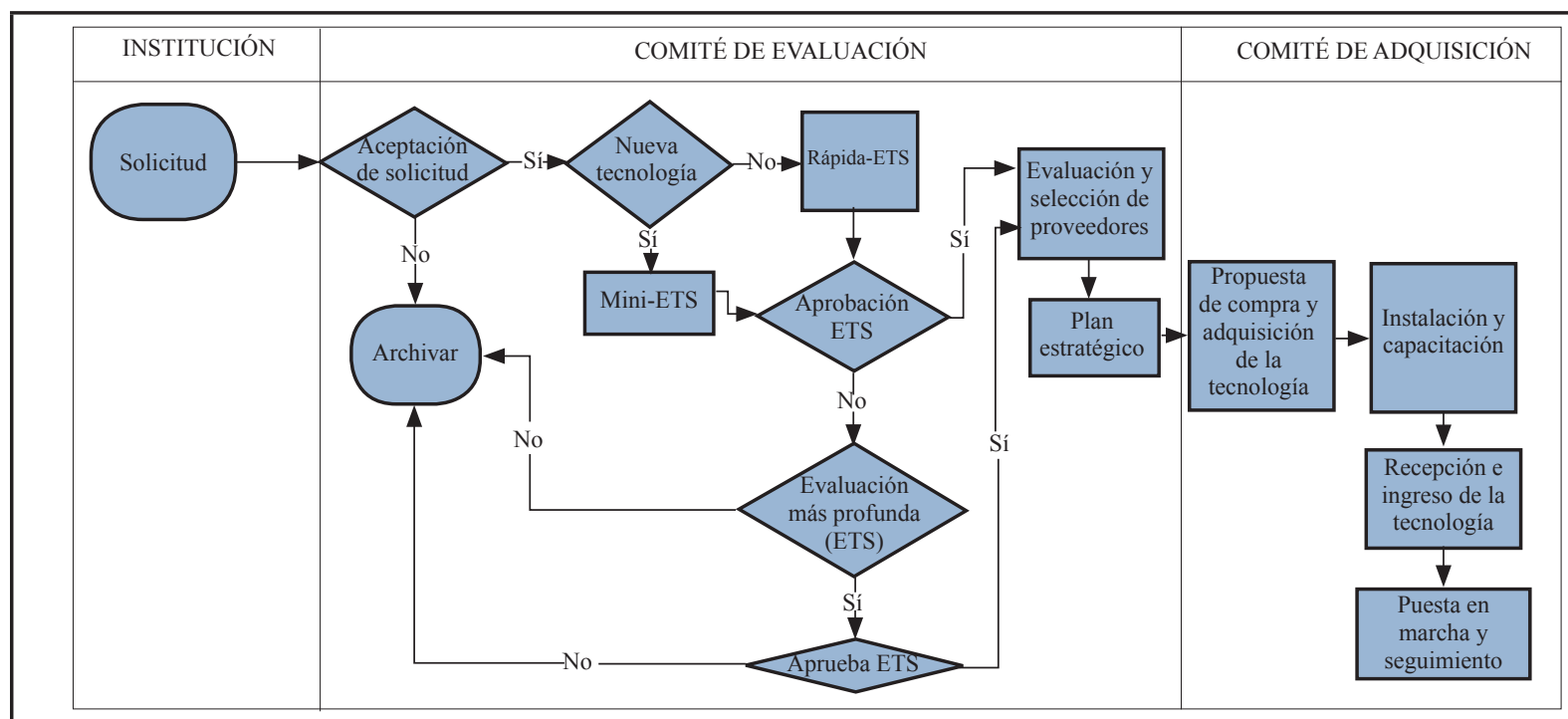

Fig. 1. Método de incorporación estratégica de tecnología biomédica

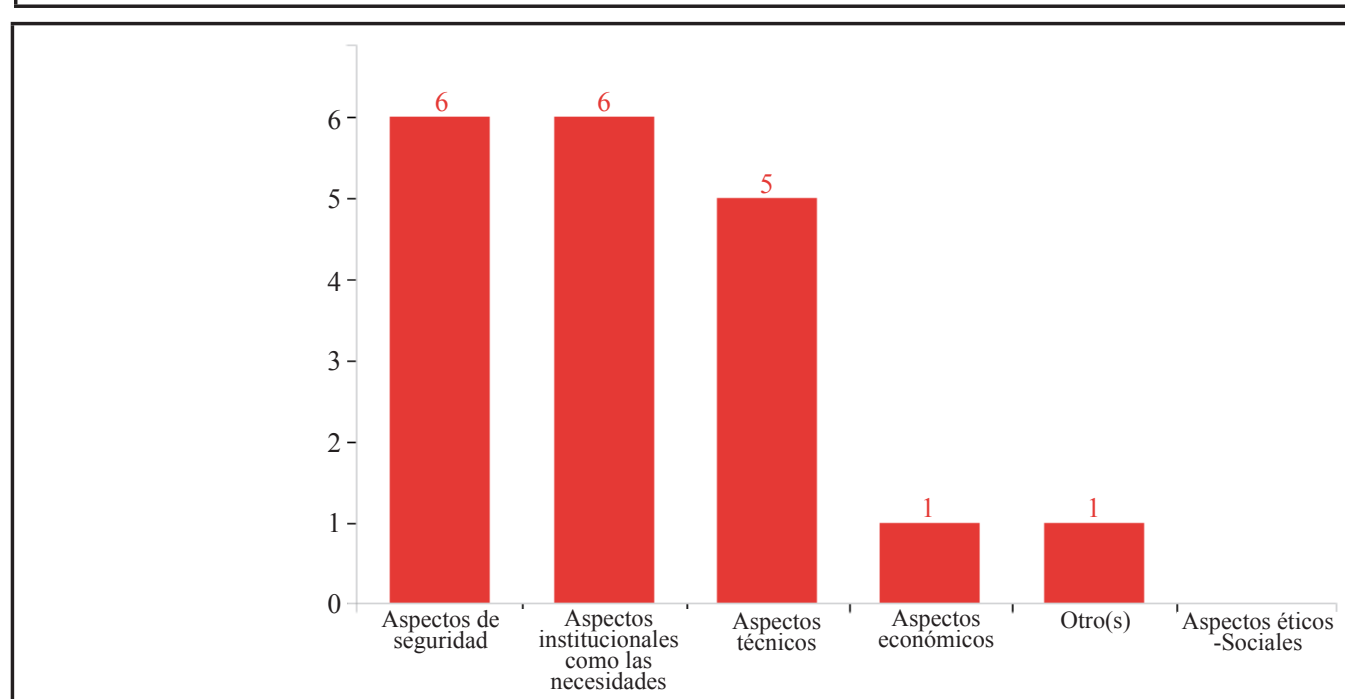

Fig. 2. Nivel de importancia de criterios definidos por los IC en la encuesta 
Aspectos tales como compatibilidad con otros equipos de la institución, respaldo en capacitaciones, opiniones de los futuros usuarios y facilidad en limpieza y desinfección, son factores que también tienen en cuenta.

\section{B. Construcción del método}

La Fig 1. ilustra el método desarrollado. A través de un flujograma se describe la secuencia del método, esto ofrece una idea general de los pasos que se deben seguir y permite el desarrollo organizado del método propuesto.

El método propuesto fue diseñado con base a las consideraciones que expresaron los IC en la encuesta, además respeta los conceptos de planeación, adquisición y gestión, y permite articularse con las políticas y planes estratégicos de la institución de salud que desee implementarlo $[4,5]$.

Para aplicar correctamente el método, es importante que la institución sea de tercer nivel y cuente con las siguientes características:

- Un comité de necesidades que se encargue de realizar análisis interno y externo [5].

- Un software que permita almacenar el inventario de los equipos biomédicos de la institución con las hojas de vida, historial de mantenimientos y repuestos usados, estado de funcionamiento, historial de eventos adversos.

- Un comité de evaluación con las facultades para recibir, analizar y evaluar las solicitudes de tecnologías de todos los servicios asistenciales [6].

- Un comité de adquisición encargado de realizar el proceso de compra, recepción, instalación, puesta en marcha y seguimiento de la tecnología.

Dependiendo del tamaño y número de equipos médicos de la institución, el comité de necesidades debe estar conformado por dos ingenieros biomédicos, uno encargado de identificar las necesidades internas de la institución y otro para identificar las necesidades externas (se recomienda que esta persona esté dedicada exclusivamente a estas tareas) [5].

El comité se debe reunir continuamente para evaluar tecnologías nuevas y emergentes que ya están en el mercado para la identificación de futuras adquisiciones; adicionalmente deben analizar qué equipos necesitan ser renovados o actualizados. Al realizar el autodiagnóstico deberá tener en cuenta tanto la situación tecnológica como las señales que se reciben del entorno a través de la vigilancia tecnológica [7].

El comité de evaluación es una asociación temporal durante el proceso de incorporación. El comité es un grupo multidisciplinario, representando diferentes perspectivas que realizan un análisis de las evidencias y recomendaciones útiles para toda la estructura hospitalaria [8]. Se recomienda que este comité esté conformado por un IC, un representante de finanzas y un arquitecto, de ser necesario. Adicional a esto se debe consultar la opinión del personal asistencial o de un médico de la especialidad donde se va requerir la tecnología, una enfermera jefe del área y personal que vaya a manejar el equipo [9].

El IC, dentro de sus funciones en el comité, debe encargarse de la gestión de las instalaciones: impacto del ambiente y requisitos de los servicios públicos; información de la tecnología: software de soporte y problemas de red; gestión de materiales: suministros, accesorios, vendedores alternativos, equipo y mobiliario auxiliar, fuentes alternativas de suministros y servicios. Información de la salud: datos epidemiológicos y regulaciones; manufactura: especificaciones del producto (ficha técnica), instalación, requerimientos operacionales y garantía [8].

El IC debe determinar la verdadera necesidad de adquisición de la tecnología, analizar consideraciones especiales de seguridad y rendimiento, seleccionar el producto a evaluar, escribir las especificaciones de compra, dirigir la evaluación de los productos, la selección del producto final y conducir la evaluación del rendimiento una vez que el producto fue puesto en marcha [6].

El método permite identificar las necesidades tanto externas como internas de la institución, priorizar las solicitudes de adquisición, determinar qué tipo de tecnologías son viables para adquirir con base en los resultados de la evaluación, y sugiere cuál proveedor, marca y modelo son los más indicados según las especificaciones técnicas, costos y servicios que ofrecen cada uno.

El método propuesto se estructura con base en un proceso continuo de IT que vincula cinco (5) tipos de evaluaciones las cuales se describen a continuación:

Evaluación de Necesidades: este primer proceso inicia con la implementación de una cultura de reporte de necesidades reales por parte de los servicios asistenciales, diferenciando siempre necesidades y deseos, evitando desde un principio la posibilidad de adquirir equipos que en un futuro podrían quedar sub utilizados o posibles sobre costos por compra de accesorios y consumibles [2].

El proceso incluye el concepto de vigilancia tecnológica como fuente de evaluación de necesidades externas, donde se identifique tecnologías nuevas y emergentes que se encuentren en el mercado para planear futuras adquisiciones basándose en los servicios de salud que se desean prestar [3]. Estas necesidades externas se articulan con las tendencias epidemiológicas que reportan organismos nacionales y regionales; además permite analizar las 
capacidades tecnológicas de la institución con base en los resultados de una evaluación de las necesidades internas. En esta se debe realizar un inventario de los equipos para identificar cuáles son las tecnologías existentes en la institución, cuáles tienen posibilidad ser cambiadas, cómo se realizaría este cambio y qué costo tendría. De esta forma se podría hacer una panificación de lo que se adquirirá a corto y mediano plazo [5].

Evaluación de Seguridad: en este punto, el método contempla los riesgos y el historial de recalls asociados a un equipo antes de tomar una decisión de compra [6].

Esta evaluación puede tener dos enfoques, el primero solo tiene en cuenta el riesgo inminente de un dispositivo según su complejidad y forma de uso, considera la probabilidad de falla durante su uso. El segundo cuantifica la probabilidad que nace de experiencias del pasado, y es recolectada por medio de búsqueda de información en bases de datos, agencias o instituciones como la FDA, ECRI y el INVIMA, entre otros organismos de evaluación y certificación [7].

Evaluación de Beneficios: aquí se consideran los posibles beneficios, directos e indirectos, que el equipo en consideración pueda traer a la institución. Ejemplos típicos de beneficios son la reducción de muertes o enfermedades, ganancia en expectativa o calidad de vida, incremento en la efectividad, productividad (reducción de los tempos de procedimiento) o confiabilidad [2].

Durante la evaluación no deben ser ignorados los beneficios indirectos, los cuales pueden ayudar a tomar la decisión final de adquirir o no un equipo. Algunos beneficios indirectos pueden ser: aumento de la productividad del usuario debido a la satisfacción que siente de trabajar con nueva tecnología o con una mejor interfaz de usuario (factor psicológico), ingresos adicionales a otros procedimientos consecuencia de esta nueva tecnología, más cobertura y mejora en el acceso. Dependiendo de la relevancia, también se debe analizar los beneficios en el impacto a la marca o prestigio de la institución, a eso se asocian variables de mercadeo y competitividad, como por ejemplo contratar médicos y cirujanos reconocidos, competitividad en el mercado o mayores tasas de ganancias [3].

Evaluación Financiera: los beneficios financieros asociados a la adquisición de nuevas tecnologías se ven reflejados en el incremento de ingresos (incremento en la productividad, aumento de las tasas de ganancia, etc.), en la disminución de los gastos (menos costos de mantenimientos, menor probabilidad de riesgos, menor tiempo de estancia del paciente y reducciones con respecto a otras instituciones, etc.) $[3,4]$.
La evaluación financiera contempla resolver cuestiones referentes a cuánto se está dispuesto a invertir para generar un producto de salud diferenciador frente a la oferta del mercado, y cómo las aseguradoras estarán dispuestas a negociar y a pagar por estos. El método sugiere además, realizar siempre un análisis del costo versus el beneficio, comparando las diferentes alternativas tecnológicas propuestas por los proveedores, identificando en este caso todos los posibles beneficios que en términos económicos puedan impactar las finanzas de la IPS [7].

El método propone otro factor económico importante para analizar y es la curva de aprendizaje en los usuarios como resultado de la IT. La necesidad de entrenamiento de los fisioterapeutas, enfermeras, médicos, y demás involucrados en el uso de la tecnología es un factor fundamental. A menudo estos profesionales necesitan realizar chequeos antes de su uso, intervenciones de emergencia ante una falla del equipo y entender las limitaciones de la tecnología [3].

Evaluación Ética y Social: con esta evaluación se pretende establecer qué impacto a la sociedad tendría la IT y el uso de esta. Por lo tanto, el método propone contemplar aspectos éticos que puedan influenciar la decisión de incorporar o no la nueva tecnología [7].

Por lo tanto se deben estudiar las disposiciones legales que afectan la adquisición, la accesibilidad de la tecnología, el cumplimiento de los derechos humanos, la integridad y dignidad humana, los posibles conflictos con convicciones religiosas y culturales y la estigmatización y discriminación [7].

Dentro de la evaluación ética de la tecnología también se deben tener en cuenta factores como si para realizar un procedimiento médico quirúrgico se necesita la firma de un consentimiento informado para situaciones especiales.

Adquisición: después de realizar los diferentes procesos de evaluación de la tecnología, el método propone realizar la etapa de la adquisición, allí se articula con los procedimientos establecidos por las IPS en donde se obtiene lo que se requiere según lo planeado.

Para esta etapa el método propone implementar sesiones de socialización y participación de profesionales de diferentes disciplinas, por ejemplo escuchar la opinión tanto de los médicos especialistas que harán uso de la tecnología como de los ingenieros biomédicos que tienen la perspectiva de cómo la tecnología será usada, operada, y los principales aspectos que se deben tener en cuenta en cada tipo de tecnología [4].

Las actividades realizadas durante esa etapa deben ser constantemente supervisadas y no relegadas a una sola área de la institución, esto debido a que hay numerosas decisiones de carácter crítico que deben ser tomadas durante la adquisi- 
ción, decisiones que requieren de personas con conocimiento sólido y claro, entendimiento de la misión y los objetivos que se desean alcanzar al realizar la adquisición [3].

Selección: Para el proceso de selección, el método propone recolectar bajo un formato específico, la misma información proveniente de diferentes proveedores. El siguiente paso es comparar un producto con otro para identificar aquella tecnología que más se ajusta a los beneficios y consideraciones generadas en las evaluaciones previas [6].

Las especificaciones, típicamente, se agrupan en categorías generales que incluyen: desempeño en general, seguridad, facilidad de uso y otros factores relacionados con el usuario, calidad de construcción, servicio y el costo en general. Las categorías no tienen un peso igual sobre la decisión final y habrá unas a las que se les tenga que poner más atención que a otras [6].

Cuando se llega a la etapa de comparar los costos de las diferentes opciones que se están evaluando, deben ser considerados todos los costos que implica poseer la tecnología durante su vida útil para que la evaluación financiera de cada uno de los productos quede completa [6].

Debido a que el costo después de la compra está entre 2-5 veces el costo de adquisición, el método propone la importancia de evaluar todos los costos antes de realizar una compra [3].

Proceso de compra: en este paso se recogen los documentos elaborados en el proceso de planeación como: justificación de la tecnología, las ETES, el resultado de la evaluación de las propuestas y el plan estratégico. El resultado de la evaluación de las propuestas debe indicar claramente todas las prestaciones que ofrece el proveedor seleccionado, en cada uno de las características técnicas como mantenimiento, repuestos, etc.

En esta fase el método sugiere redactar el contrato, definiendo la modalidad y condiciones de entrega del equipo. Una vez firmado el contrato se procede con la compra. Se debe enviar al proveedor el listado de los documentos necesarios con los que debe entregarse el equipo en la recepción.

Recepción e instalación: se recibe la tecnología, se verifica que los documentos que se describen estén en regla y que el equipo esté en óptimas condiciones. Para esto se realiza un test de funcionamiento. Se recomienda que este proceso de recepción se efectúe en el área de ingeniería.

Se debe utilizar el traslado del equipo al servicio, realizar la instalación del equipo en el servicio, verificando nuevamente las pruebas de funcionamiento y seguridad. Una vez aprobado esto se firma el recibo de satisfacción por el jefe de área.
Entrenamiento: realizar entrenamientos según el cronograma pactado en la solicitud, tanto al personal de ingeniería como al asistencial, garantizando completo entendimiento en el uso de la tecnología. Todo el personal que va a quedar encargado del uso de la tecnología, debe quedar satisfecho con la información brindada. Todas las capacitaciones se deben realizar antes de la puesta en marcha del equipo [12].

Puesta en marcha y seguimiento: se autoriza al servicio utilizar el equipo cuando sea requerido. Se recomienda realizar un seguimiento al funcionamiento para garantizar que se encuentre en óptimas condiciones.

Se realiza un seguimiento a la tecnología una vez instalada y puesta en marcha, esto con el fin de comprobar si se atiende la demanda esperada, las características técnicas ofrecidas, si se suplen las necesidades y se generan los beneficios que se identificaron en la evaluación de la adquisición.

Este paso se debe realizar definiendo y monitoreando indicadores que debe definir la institución según sus necesidades, y la información que deseen obtener de este seguimiento. De esta etapa de debe generar una retroalimentación a todo el proceso de IT en general, como al ajuste de los planes estratégicos y presupuestos de la institución.

\section{Aplicación y validación}

Para este punto se aplicó el método en un caso de estudio de IT de dos equipos médicos en una IPS de la ciudad de Cali, Colombia. La IPS pretendía adquirir un equipo por renovación tecnológica y otro considerado nuevo. La validación se realizó a través de la consulta a dos expertos de la IPS seleccionada; allí se consideraron variables de usabilidad y calidad de la información que entrega el método para la toma de decisiones.

\section{- $\quad$ Caso de estudio}

La validación se realizó en la Clínica de Occidente de Cali, y los equipos seleccionados fueron: un acelerador lineal como nueva tecnología y una autoclave como renovación.

El método ayudó a identificar factores claves para la toma de decisiones, las cuales se resumen en la Tabla 1.

A cada una de las tecnologías se le realizó todo el proceso de incorporación diseñado, diligenciando para cada uno los formatos correspondientes, cada una de las etapas se evaluaron con base a puntajes y al finalizar se definió el proveedor más indicado según las especificaciones técnicas y las condiciones ofrecidas por los diferentes proveedores evaluados. 
Tabla 1. Factores claves para la toma de decisiones

\begin{tabular}{|c|c|}
\hline Acelerador Lineal & Autoclave \\
\hline $\begin{array}{c}\text { Se estima atender el } 33,33 \% \text { de } \\
\text { la población con cáncer en el } \\
\text { Valle del Cauca }\end{array}$ & $\begin{array}{l}\text { La capacidad instalada no } \\
\text { satisface las necesidades }\end{array}$ \\
\hline $\begin{array}{l}\text { Aumento de la eficiencia y } \\
\text { calidad en la atención del } \\
\text { servicio de radioterapia }\end{array}$ & $\begin{array}{l}\text { Aumento de la eficiencia y } \\
\text { calidad en la atención de los } \\
\text { servicios asistenciales }\end{array}$ \\
\hline $\begin{array}{l}\text { Disminución de la estancia de } \\
\text { pacientes }\end{array}$ & $\begin{array}{c}\text { El valor de los repuestos } \\
\text { invertidos actualmente supera el } \\
100 \% \text { del costo del equipo }\end{array}$ \\
\hline $\begin{array}{l}\text { Uso del equipo: de } 20 \text { a } 50 \text { veces } \\
\text { por semana }\end{array}$ & $\begin{array}{l}\text { Uso del equipo: más de } 50 \text { veces } \\
\text { por semana }\end{array}$ \\
\hline $\begin{array}{l}\text { Se requieren adecuaciones } \\
\text { infraestructuras especiales. De } \\
\text { alto costo e impacto para la IPS }\end{array}$ & $\begin{array}{l}\text { No requiere de adecuaciones } \\
\text { especiales }\end{array}$ \\
\hline $\begin{array}{c}\text { Su IT se estima de mediano a } \\
\text { largo plazo }\end{array}$ & Su IT se estima a corto plazo \\
\hline
\end{tabular}

\section{Validación con expertos}

Los resultados obtenidos de la implementación del método se sometieron a evaluación por dos expertos de la Clínica de occidente, a través del diligenciamiento de un formato de preguntas. Los dos expertos fueron el coordinador del área de ingeniería, encargado de la gestión de la tecnología de la institución, y el gerente operativo cuya función es la de gestionar las tecnologías de alto costo, como el acelerador lineal.

La validación del método fue exitosa, tuvo un visto bueno por parte de los expertos y lo consideran bien estructurado y de gran ayuda para una toma de decisiones efectiva y de calidad.

En general, los resultados superaron las expectativas iniciales, los expertos manifestaron estar satisfechos con la el proceso que describe el método, la forma en que se presentan los resultados y los informes arrojados para cada una de las tecnologías. Se evidencia un deseo por emplear la información suministrada, por el método para tomar la decisión final y por la ayuda que representa esta herramienta para los procesos de IT biomédica en las IPS.

\section{DisCUSIÓN}

Se encontró variedad de artículos relacionados con la IT que formulan los aspectos más importantes que se deben evaluar antes de adquirir una tecnología. Sin embargo la mayoría de los métodos que se encuentran en la literatura son muy generales y arrojan resultados cualitativos, por lo cual el método propuesto genera valor agregado al generar datos cuantitativos que permiten estructurar una toma de decisión más acertada.
El análisis de los costos invisibles es un aspecto crítico cuando se va a evaluar un equipo, y por lo general no son considerados al momento de compra. Hay evidencia de que costos invisibles son incluso mayores al valor del equipo, por lo que puede suceder que la institución no se encuentre en condiciones económicas para cubrir estos costos durante la vida útil de la tecnología.

La viabilidad del método se comprobó mediante su aplicación en el proceso de incorporación de un acelerador lineal y una autoclave en la Clínica de Occidente. Se confirmó que este plantea un adecuado sistema para identificar las necesidades tanto externas como internas de la institución, ayuda a priorizar las solicitudes de adquisición, determina qué tipo de tecnologías son viables para adquirir y con base en los resultados de la evaluación sugiere cuál proveedor, marca y modelo son los más indicados según las especificaciones técnicas, costos y servicios que ofrece cada uno.

La aplicación del método permitió concluir que el tiempo de garantía es un factor de alto peso en el costo total de la vida útil del equipo, debido a que en los años de garantía no se generan gastos de repuestos y mantenimiento. Con base a esto se le recomienda a la Clínica de Occidente, en el caso de adquirir el acelerador lineal, solicitar extender el tiempo de la garantía por ser una tecnología de alta inversión a corto y largo plazo.

Se identificó que la compra de un equipo no se puede basar en el precio de este, porque la tecnología seleccionada puede no ser la más económica pero ofrecer mejores especificaciones o servicios post venta, como se mostró en el caso del acelerador lineal, donde la tecnología seleccionada es más costosa pero ofrece servicios que la hacen mejor opción que la de otro proveedor.

De la validación con los expertos se concluye que, aunque es un método bien estructurado para la adecuada incorporación de tecnología, no alcanza la excelencia y se debe mejorar en aspectos como claridad en el contenido y cantidad de criterios evaluados para que sea más factible su aplicación en las instituciones de salud.

En general, consideran que es una herramienta que ayuda a la toma de decisiones ya que permite aclarar dudas, comparar y seleccionar la mejor opción. Ambos expertos manifestaron interés en utilizar la información generada por la herramienta para tomar la decisión final de adquisición.

\section{CONCLUSIONES}

El método desarrollado guía al usuario para realizar todo el proceso de incorporación de una manera sencilla y sistemática. 
Ayuda a las instituciones a identificar las necesidades evaluando el estado de la tecnología con la que se cuenta y las condiciones médicas más relevantes en la región, para luego, con base en las políticas y metas que desea alcanzar la institución, decidir cuál es la tecnología más apropiada para cubrir dichas necesidades.

Propone un proceso estandarizado para la toma de decisiones objetivas ayudando a la organización de procesos y procedimientos de la institución, que contribuye al cumplimiento de los estándares de calidad el cual es un tema esencial en IPS de tercer nivel.

Para la aplicación del método la institución debe ser de tercer nivel y contar con un software para gestionar el inventario y comités de necesidades, de evaluación y adquisición. Esto es debido a que el método fue desarrollado asumiendo que la institución contaba con estas características, y si la institución no cumple con esto, la aplicación del método se dificultará.

El método es válido para incorporar estratégicamente tecnología biomédica en una institución de salud porque sugiere qué decisión tomar en cada etapa del proceso; la prueba de esto es la verificación con expertos y su retroalimentación al validar el método y la herramienta.

Los objetivos se cumplieron satisfactoriamente y se superaron al aplicar el método en un caso real del análisis de incorporación de dos tecnologías biomédicas en la Clínica de Occidente en la ciudad de Cali. Se logró emplear tantos los formatos para incorporar una tecnología nueva con el acelerador lineal, como los de incorporación de tecnologías ya existentes en la institución con la autoclave.

\section{REFERENCIAS}

[1]. Castañeda C., Fonseca M., Núñez J., Zapata J.; Gonzalo J. La Sostenibilidad Financiera del Sistema de Salud Colombiano: Dinámica del gasto y principales retos de cara al futuro, Bogotá: Fedesarrollo, 2012

[2]. Organización Mundial de la Salud. Resolución WHA60.29. (julio de 2007). Consultado el 15 de noviembre de 2016 en: http://www. who.int/medical_devices/policies/resolution_wha60_r29-sp.pdf.

[3]. Wang B. Strategic Health Technology Incorporation, Organ \& Claypool, 2009.

[4]. Aparecido Nunes A., Marques de Mello L., Wichert Ana L., Mazzoncini de Azevedo Marques P., Lessa Dallora M. E., Zangiacomi Martinez E., Pazin Filho A., Barbosa Coelho E. Avaliação e incorporação de tecnologias em saúde: processo e metodologia adotados por um hospital universitário de alta complexidade assistencial. Cadernos de Saúde Pública, 29(1), 179-186, 2013.

[5]. Santos F.A., Margotti A.E., Ferreira F.B., Garcia R. Health Technology Assessment Applied to Health Technology Management through Clinical Engineering. IFMBE Proceedings, 41, 1104-1107, 2013.
[6]. Organización Mundial de la Salud. Guía de recursos para el proceso de adquisición, Ginebra: OMS, 2012.

[7]. Paz F.A., Del Valle Made E., Hadad Salomón R. Ethical and legal model for technological surveillance system. Interactive Collaborative Learning (ICL), 999-1006, 2015.

[8]. Carvajal Tejada M.; Ruiz Ibáñez C.G. Evaluación técnica y clínica de tecnología biomédica en procesos de adquisición: un enfoque en evaluación de tecnologías en salud. Revista Ingeniería Biomédica, 2(4), 34-45, 2008..

[9]. Calil S. J. Caminhos para a incorporação de tecnologias em saúde. Revista Debates GV saúde, 3, 31- 34, 2007.

[10]. Atles L. A practicum for Biomedical Engineering \& Technology Management Issues, Kendall/Hunt Publishing Company, 2008.

[11]. Gonzáles D. Herramienta de evaluación para adquisición de equipos biomédicos. Medellín. 2014.

[12]. Van Hove P., van Wijk R., Verdaasdonk E., Stassen L., Dankelman J. Training and assessment of equipment-related competence. Comparison of a petrochemical company and a hospital. Health technology, 3, 221-226, 2013. 